\title{
CONF-9704102--2 \\ RF Heating experiments in CHS and RF development for LHD
}

T. WATARI, R. KUMAZAWA, K. NISHIMURA, T. MUTOH, T. SEKI, S. MASUDA I, F. SMMBO, T. DDO2, R. AKIYAMA, A. ANDO, A. EJIRI, H. IDEI, K. IDA, H. IGUCHI, A. FUJISAWA, M. ISOBE', M. IWASE!, S. KUBO, K. MATSUOKA, T. MORISAKI, S. MORITA, S. MUTOH, S. MURAKAMI, S. OKAMURA, T. OZAKI, S. SAKAKIBARA, M. SASAO, C. TAKAHASHI, T. KAWAMOTO, K. TANAKA, J. XU, H. YAMADA, I. YAMADA, J.M. NOTERDAEME3

National Institute for Fusion Science

1Department of Fusion Science, The Graduate University for Advanced Studies

2Department of Energy Engineering and Science, Nagoya University

Furo-cho, Chikusa-ku, Nagoya, 464-01, Japan

3Max-Planck Institute for Plasma Physics, Garching, Germany

D.A. RASMUSSEN, J.F. LYON, J.B. WILGEN, D.E. GREENWOOD, D.J. HOFFMAN, E.F. JAEGER, M. MURAKAMI

Oak Ridge National Laboratory

Oak Ridge, TN 37831, U. S. A.

\section{Introduction}

The Large Helical Device, LHD, is in its final construction phase. It is a $l=2, m=10$ Heliotron/Torsatron type helical system with a major radius of $4 \mathrm{~m}$. The compact helical system, CHS, is a $l=2, m=8$ helical system of the same type with a major radius of $1 \mathrm{~m}$. CHS has been used for supporting experiments to clarify physics issues of helical systems and to examine the key ideas which will be applied to LHD. This paper summarizes the experimental results of those supporting experiments in CHS and how this knowledge is incorporated in the design of RF heating in LHD. ICRF Heating results in CHS by use of loop antennas are described in section III. The results of using a Nagoya type-III coil is described in Section III. Two types of antennas used in the initial phase of the LHD ICRF Heating: a loop antenna designed for steady state heating and a folded wave guide antenna designed for $\mathbb{I B W}$, are described in section $\Gamma$.

\section{ICRF Results in CHS}

To judge the chances of success of ICRF Heating in LHD, we needed to assess experimentally the applicability of ICRF heating in a helical system, particularly in CHS which has similar configuration. In tokamak experiments, the impurity problem which has been the largest issue of ICRF heating, was finally solved and 


\section{DISCLAIMER}

This report was prepared as an account of work sponsored by an agency of the United States Government. Neither the United States Government nor any agency thereof, nor any of their employees, make any warranty, express or implied, or assumes any legal liability or responsibility for the accuracy, completeness, or usefulness of any iniormation, apparatus, product, or process disclosed, or represents that its use would not infringe privately owned rights. Reference herein to any specific commercial product, process, or service by trade name, trademark, manufacturer, or otherwise does not necessarily constitute or imply its endorsement, recommendation, or favoring by the United States Government or any agency thereof. The views and opinions of authors expressed herein do not necessarily state or reflect those of the United States Government or any agency thereof. 


\section{DISCLAMMTR}

Portions of this document may be illegible in electronic image products. Images are produced from the best available original document. 
ICRF heating is now regarded to be a heating scheme as reliable as Neutral Beam Heating. However, in helical systems, previous ICRF heating experiments suggested that many problems have still to be solved before ICRF heating is clearly established. ICRF Heating experiments were conducted at NIFS in 1993 and in 1995. In the experiments in 1995, a total of 5 antennas were installed. Four of them, with the shape shown in Fig. 1(a), followed the 45 degree slanted ellipsoidal shape of the plasma. One of the antennas (Fig.1(b)) was put where plasma cross section is vertically elongated.The latter has the same shape as the one planned for LHD and is a developmental test of the LHD antenna.
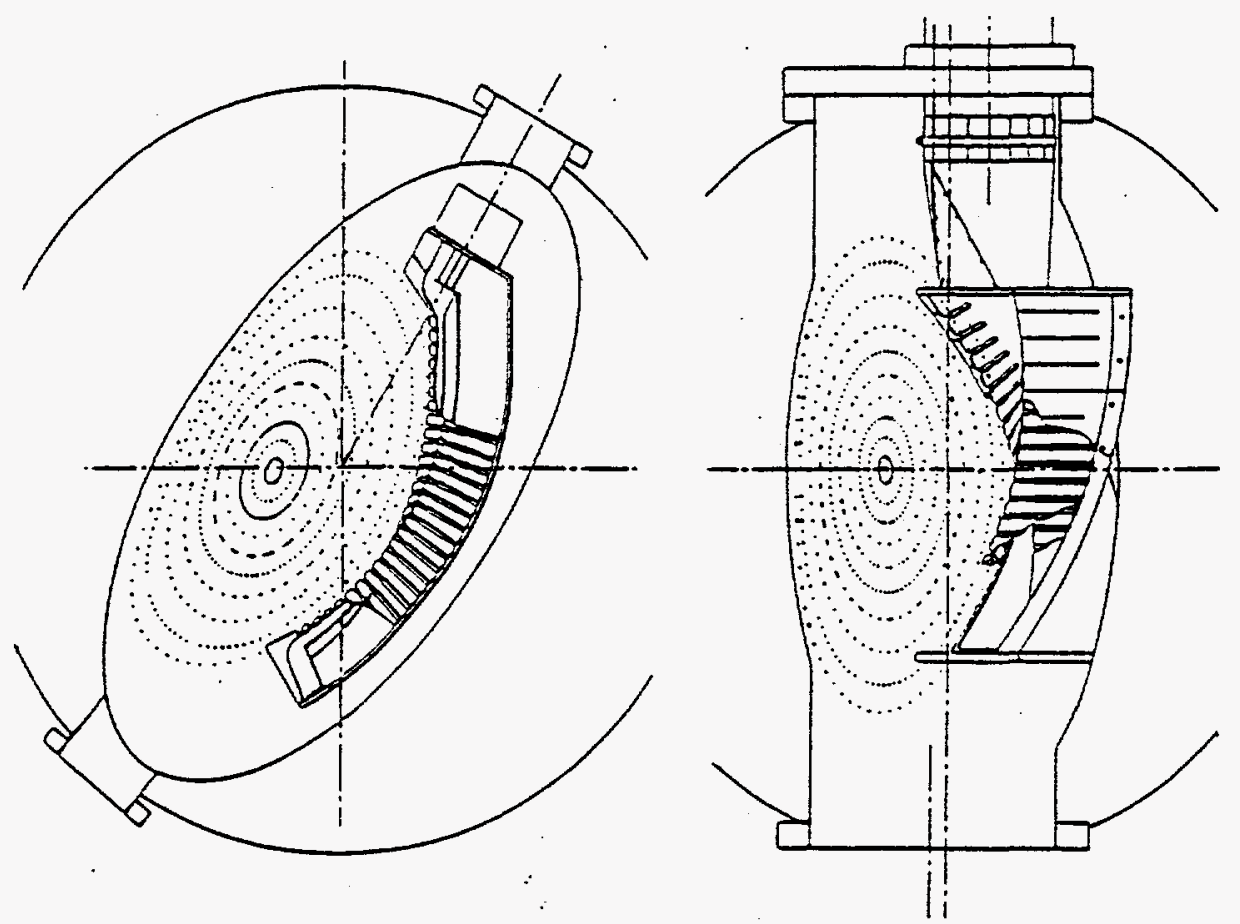

Fig. 1 Cross section of singie strap antennas installed at (a); P port, and (b); $U$ port in CHS. The current strap of the P-antenna is $4 \mathrm{~cm}$ wide and $40 \mathrm{~cm}$ long. The current strap of the $U$-antenna is $16 \mathrm{~cm}$ wide and $40 \mathrm{~cm}$ long.

ICRF heating in tokamaks are often discussed based on the vertically stratified slab model, for the wave propagation and absorption is strongly characterized by the ion cyclotron, hybrid resonance, and cutoff layers. This kind of consideration is supposed to be useful also in designing the ICRF Heating scenarios for Helical systems, since the wave itself find its way along the steepest gradient of magnetic field as it propagates displaying refraction and reflection characteristics. Therefore, we examine the wave propagation simply in terms of the local dispersion relation given by

$\mathrm{n}_{\perp^{2}}=\left(\mathrm{R}-\mathrm{n} / /^{2}\right)\left(\mathrm{L}-\mathrm{n} / /^{2}\right) /\left(\mathrm{S}-\mathrm{n} / /^{2}\right)$,

The magnitude of the magnetic field across a poloidal section in a Heliotron/Torsatron type Helical system has a saddle shaped function: it increases with minor radius along the shorter axis of the ellipse and decreases with radius 
along the longer axis. This gives a quite different arrangement of the resonance, cut off, and cyclotron layers from those in a tokamak. We show here two cases which will be referred to in the following argument. The RF frequency used in the experiments is $26 \mathrm{MHz}$ and the toroidal magnetic field is varied from 1.8 to $1.7 \mathrm{~T}$ at the center of the plasma. The labels $R, L$ and $S$ indicate $n_{/ / 2}=R$ (cutoff), $n_{1 /}{ }^{2}=L$ (cutoff) and $n_{/ 2}{ }^{2}=S$ (resonance) layers, respectively. Cyclotron resonance layers are indicated by $\omega=\omega_{\mathrm{cH}}$. The $U$ antenna is located at the larger major radius side of the plasma (at the right on the figure).
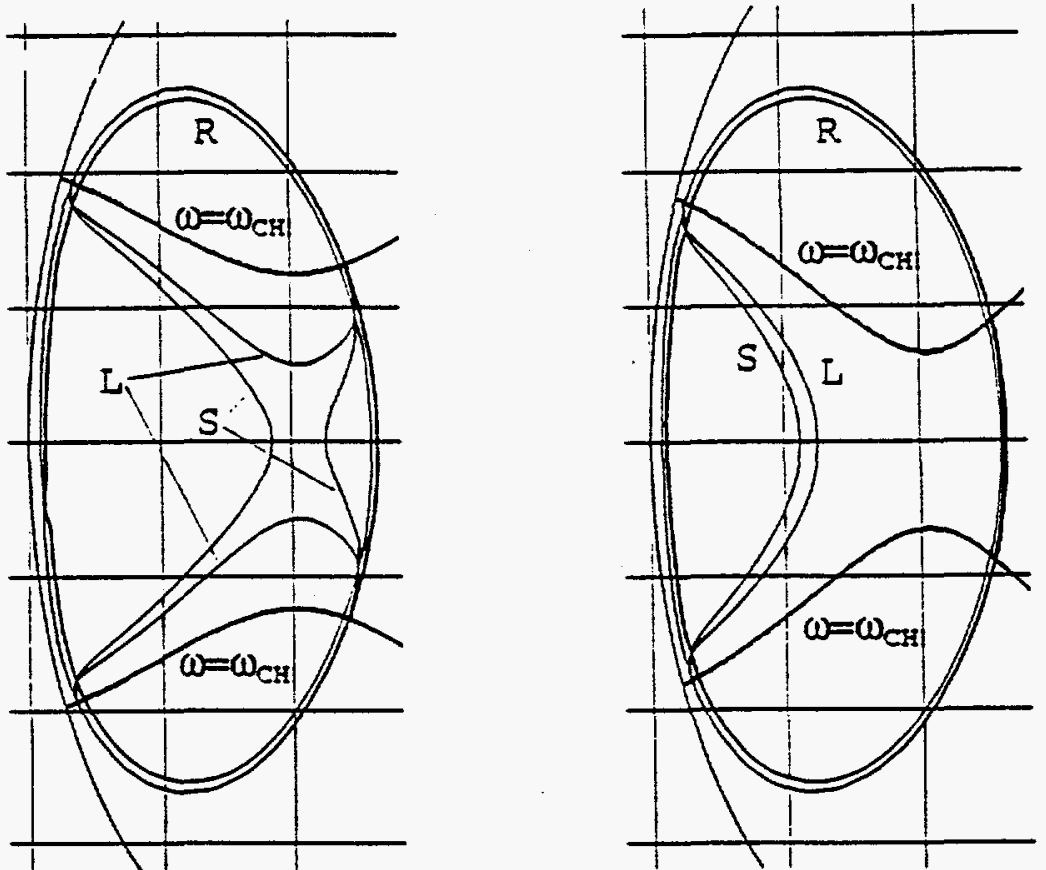

Fig. 2 Resonance and cut-off layers for the plasma cross section at the $U$ port:

(a) $\mathrm{B}_{\mathrm{T}}=1.8 \mathrm{~T}, \mathrm{n}_{\mathrm{e}}=4 \times 10^{19} \mathrm{~m}^{-3}, \mathrm{n}_{\mathrm{H}} /\left(\mathrm{n}_{\mathrm{H}}+\mathrm{n}_{\mathrm{D}}\right)=0.2$ and $\mathrm{k}_{/ /}=10 \mathrm{~m}-1$; Reference.

(b) $B_{T}=1.7 \mathrm{~T}, \mathrm{n}_{\mathrm{e}}=4 \times 10^{19} \mathrm{~m}-3, \mathrm{n}_{\mathrm{H}} /\left(\mathrm{n}_{\mathrm{H}}+\mathrm{n}_{\mathrm{D}}\right)=0.3$ and $\mathrm{k}_{/ /}=10 \mathrm{~m}-1$. The two-ion hybrid resonance layer is located at plasma center.

The case $B_{T}=1.8 T$, and $n_{H} /\left(n_{H}+n_{D}\right)=0.2$, is expected to have the best experimental conditions and is used in the reference calculation shown in Fig.(2a). The wave, launched from the antenna on the larger major radius side, approaches the ion-ion resonance layer from the high field side as illustrated in Fig.(2a). This two ion hybrid heating regime with sufficiently high minority concentration ratio is known to heat electrons. Electron heating was chosen in this experiment among various heating regimes for the following reason. If minority ions are heated, the power going to deuterium and electrons are given by Eq.(2) and Eq.(3), respectively.

$P_{H D}=3 / 2 n_{H}\left(T_{H}-T_{D}\right) / \tau_{H D} V$,

$P_{H e}=3 / 2 n_{H}\left(T_{H}-T_{e}\right) / \tau_{H e} V$.

The problem in early tokamak experiments was that the energy relaxation from 
hydrogen to bulk ions is not large enough and the power absorbed by minority ions results in the creation of a high energy tail. However, small tokamaks in the early days could not confine such energetic ions. Their loss reduced the plasma heating efficiency and further deteriorated the performance by enhancing impurity ion influx. Similar problems are anticipated for current helical devices whose confinement of fast particles is not optimal. If however, the fast wave is mode converted to the Ion Bernstein Wave and absorbed by electrons, such problem will be very much mitigated.The other key to the success of this experiment was the use of a boronized wall. Figure 3. shows the time history of a successful shot:

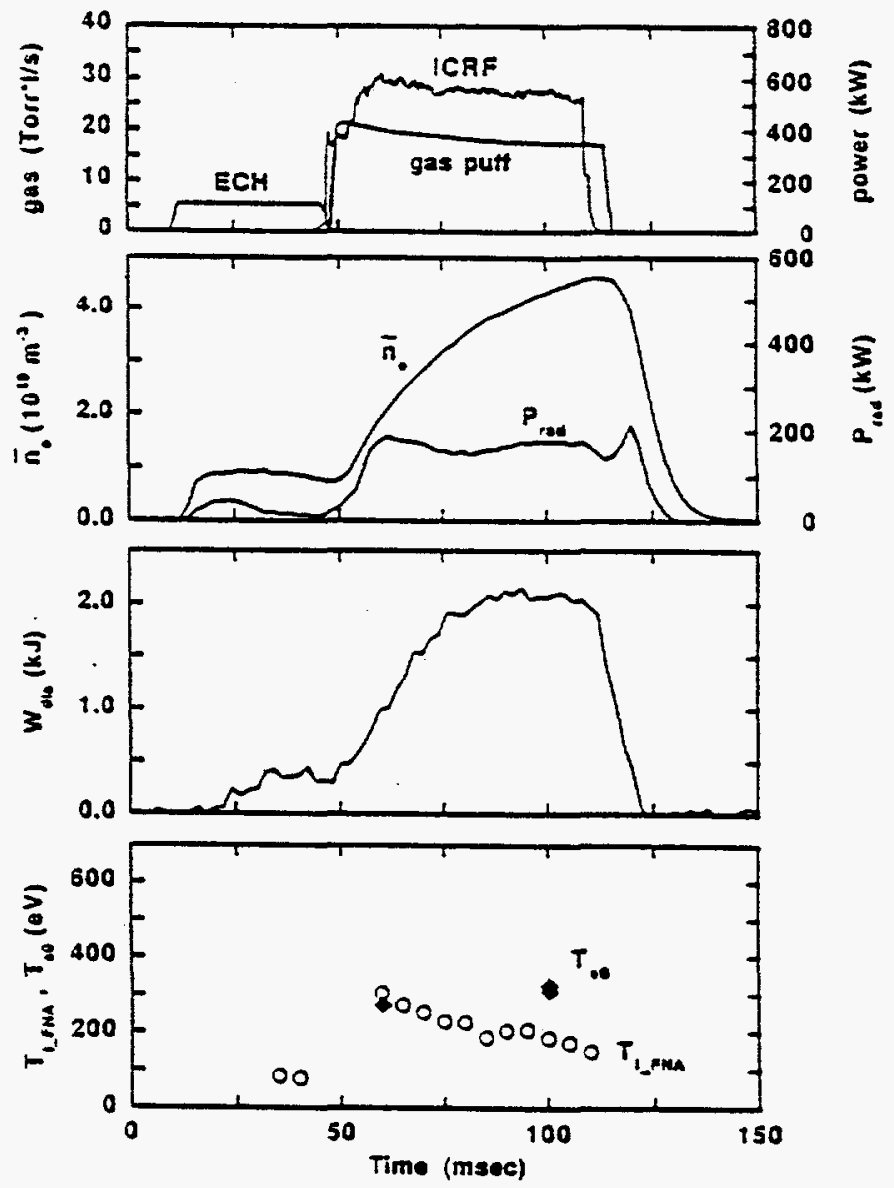

Fig. 3 Temporal evolution of plasma parameters for an ICRF heating experiment (\#40991). Top column : gas puffing rate and input power. Second column : line averaged electron density and radiation loss. Third column : stored energy measured by a diamagnetic loop. Bottom column : electron and ion temperature measured by Thomson scattering and NPA, respectively.

As it used to be in tokamak experiments, accumulation of impurities was the issue to be addressed in ICRF heating of helical systems. It is noted that the radiation loss saturated in time in this shot and the maximum stored energy is sustained. The stored energy increases to $2.2 \mathrm{~kJ}$ which is comparable to the largest stored energy 
obtained with ECH at the same power. Dependence of heating efficiency on the magnetic field and minority concentration ratio was studied in the optimization procedure. The best performance was obtained with $B_{T}-1.7 \mathrm{~T}$ and minority concentration ratio $\mathrm{n}_{\mathrm{H}} /\left(\mathrm{n}_{\mathrm{H}}+\mathrm{n}_{\mathrm{D}}\right) 0.3$, a set of parameters slightly different from our original experimental plan. Figure 3 suggests that electron heating is the dominant wave absorption mechanism since the electron temperature is clearly higher than the ion temperature at the end of the shot. We also observed the tendency that electron heating occurs preferentially when the plasma density is high. However, with this rather high minority concentration, the resonant and cutoff layers shifts as shown in Fig.(2b). Then, due to the toroidal effect, the wave should be regarded as incident from the low field side and the observed electron heating should be interpreted the other way around. Recently, there is a series of tokamak experiments of low field side launch showing electron heating. One of the models to interpret this incorpotrates interference of reflected wave with the incident wave. A preliminary examination of such mode conversion heating model gives reasonable explanation to the tendency that more portion of the wave energy is absorbed by electrons with higher plasma density.

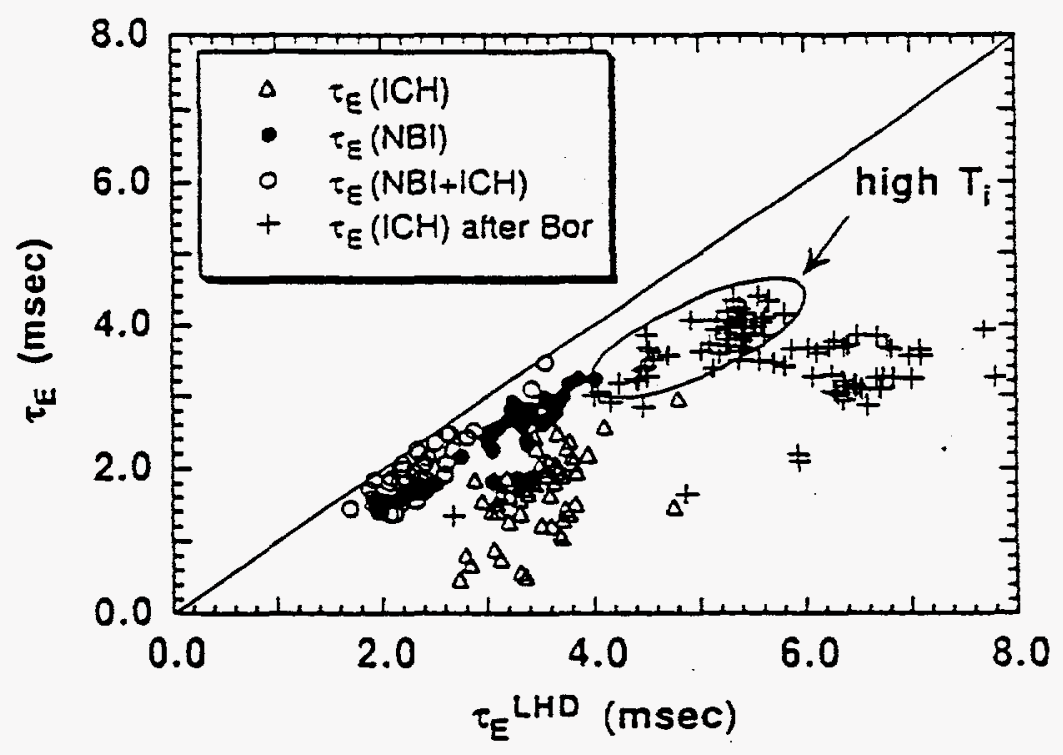

Fig. 4 Experimentally obtained energy confinement time compared to prediction of LHD scaling with corresponding plasma parameters.

Fig. 4 shows the confinement time for various experimental conditions. Many data points without boronization scatter below the prediction of LHD scaling. These includes shots where the radiation loss takes a significant part of the input power. With boronization, the impurity problem is solved and the resultant stored energy is close to the prediction of the LHD scaling. It is also noticed that shots with a combination of NBI + ICRF show performance close to or better than NBI shots. This result may be understood in terms of the nonlinear evolution of ionization stages of impurity ions. The fact that a flat top of the stored energy is obtained is encouraging from the impurity point of view. ICRF experiments are expected to be much better in LHD since it has a better alignment of the drift orbits to that of flux 
surfaces and, due to the large volume, energy relaxation is easier. Therefore, there is a possibility that even ion heating can be successfully pursued.

\section{Plasma Production by the Nagoya Type-III Coil in $\mathrm{CHS}$}

An antenna of the Nagoya type-III has current along the magnetic field lines. It has been traditionally used at Nagoya for excitation of shear Alfven wave in RFC-XX device and for excitation of the Ion Bernstein Wave in JIPP T-IIU. In CHS, it was used in the demonstration of IBWH in helical systems in the early phase of CHS experiments. The notable finding in this experiment is the ability of plasma production. In helical devices, without the Ohmic heating system, which has been the conventional way of plasma production in tokamaks, ECH plasma production has been considered indispensable as a plasma production method. Once plasma of some density is obtained, NBI is available as powerful source of plasma heating. Figure 5 shows the plasma density obtained with this antenna, as a function of the magnetic field strength for various RF frequencies at $100 \mathrm{~kW}$ power level.
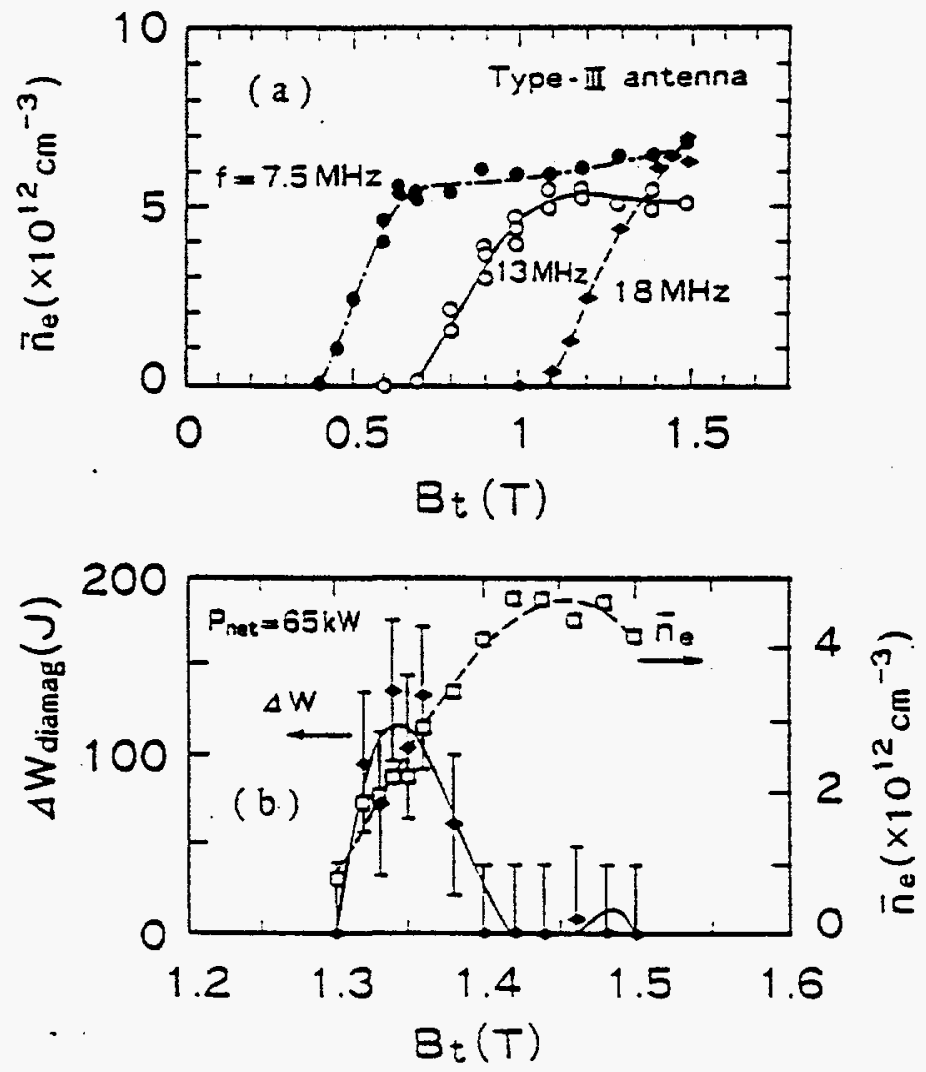

Fig.5 (a)Magnetic field dependence of the produced plasma density. (b) Magnetic field dependence of the stored energy.

Plasma is produced when the magnetic field is stronger than certain critical value, 
given by $\omega / \omega_{c}<1$ with $\omega_{c}$ the ion cyclotron frequency calculated with the magnetic field on the plasma axis. This condition is not very tight and, if the $R F$ frequency is set reasonably low, plasma production by the use of the Nagoya

type-III coil becomes independent of the magnetic field strength. This flexibility is a distinct advantage over the plasma production by ECH. This method of plasma production has been used for many experimental purposes. An important example is the high beta experiment, where lower B is preferred. Another example, shown in Fig. 6 is the investigation of the density and magnetic field dependence of the confinement time.

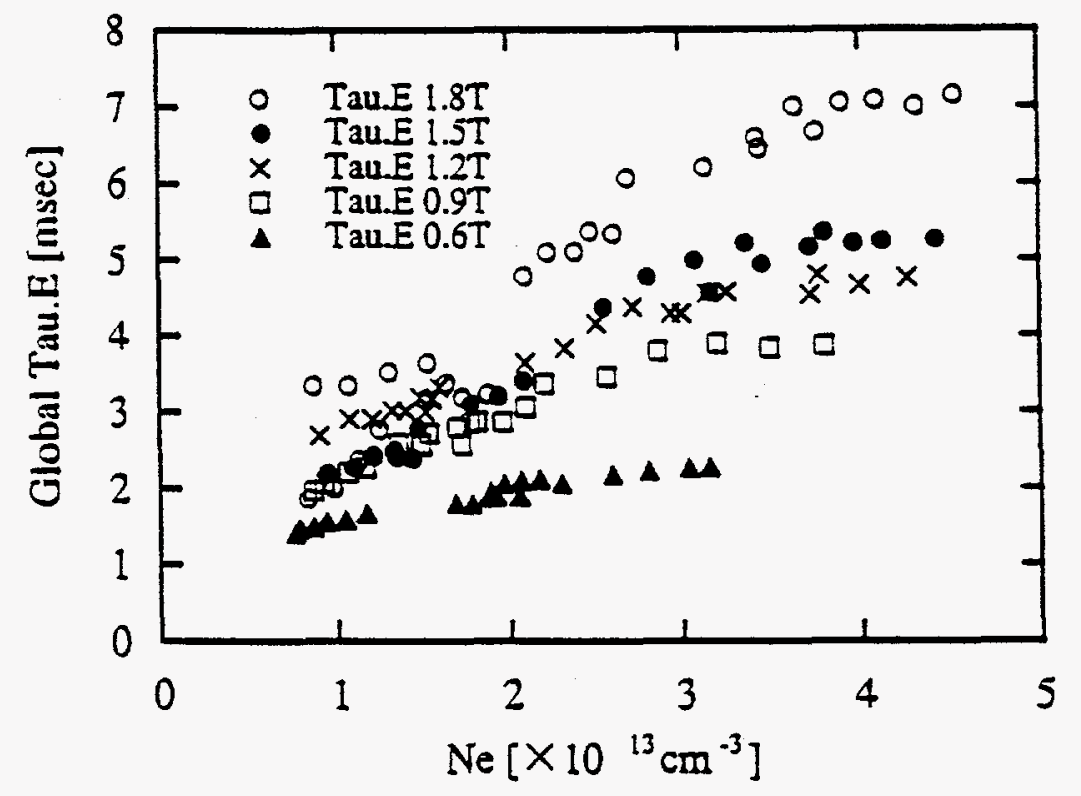

Fig.6 B dependence of the stored energy.

The type-III coil was used successfully for plasma production. In order to extend its use to plasma heating we needed to demonstrate the achievement of a certain amount of plasma energy. This was done in limited conditions: Fig5(b) shows such a case where some stored energy is obtained in the narrow window of magnetic fields. The analysis of the heating mechanism is ongoing.

\section{I.V ICRF Antennas in LHD Start up Phase}

$12 \mathrm{MW}$ ICRF heating is projected in LHD. The initial phase of the experiment starts with 3MW of ICRF heating in 1998. According to the results obtained in the CHS supporting experiment, the antenna is placed at the high field side as shown in Fig.7. The whole system is water cooled and demonstration of steady state heating is pursued, a suitable experiment for the superconducting LHD. The usefulness of the Nagoya type-III coil demonstrated in IBW/plasma production will be further pursued in LHD. In the initial phase of the experiment a wave guide antenna will be used for the same purpose as that of the Nagoya type-III coil. Their performances will be compared at an appropriate time by changing the wave guide for a Nagoya Type-III coil. Figure 8 is the artists view of the folded wave guide antenna applied 
to the LHD device.The design of these antennas is finalized and they are under fabrication in the company.

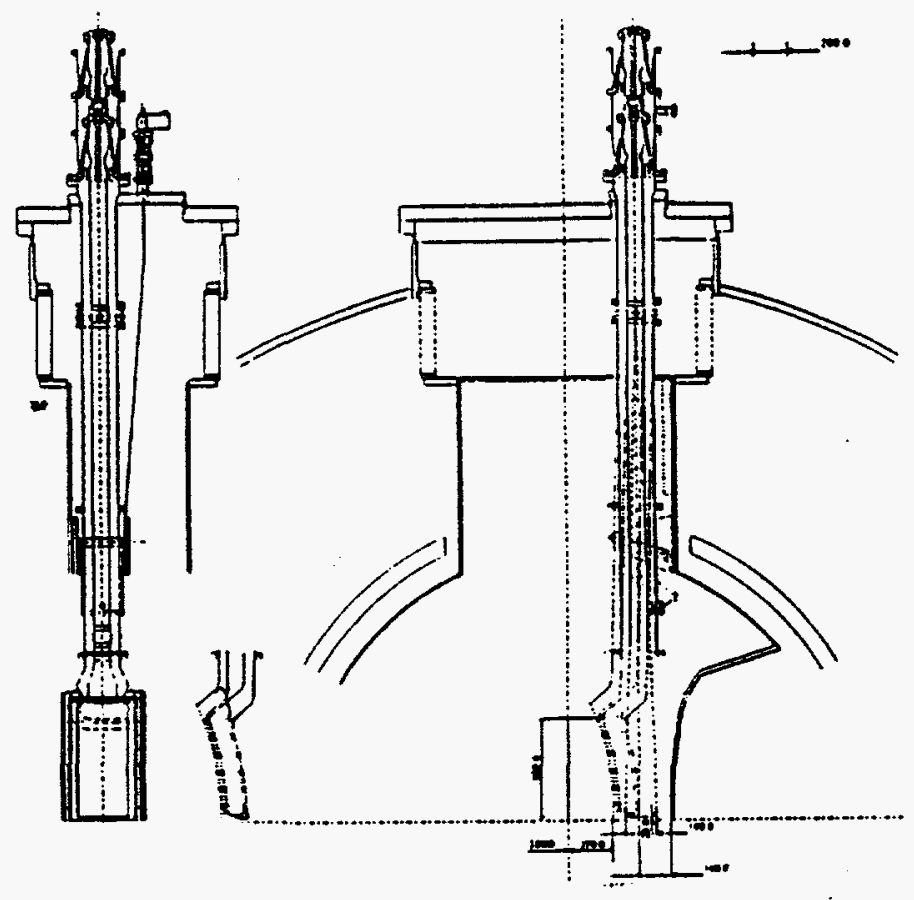

Fig7 Movable loop antenna design for LHD used for fast wave excitation

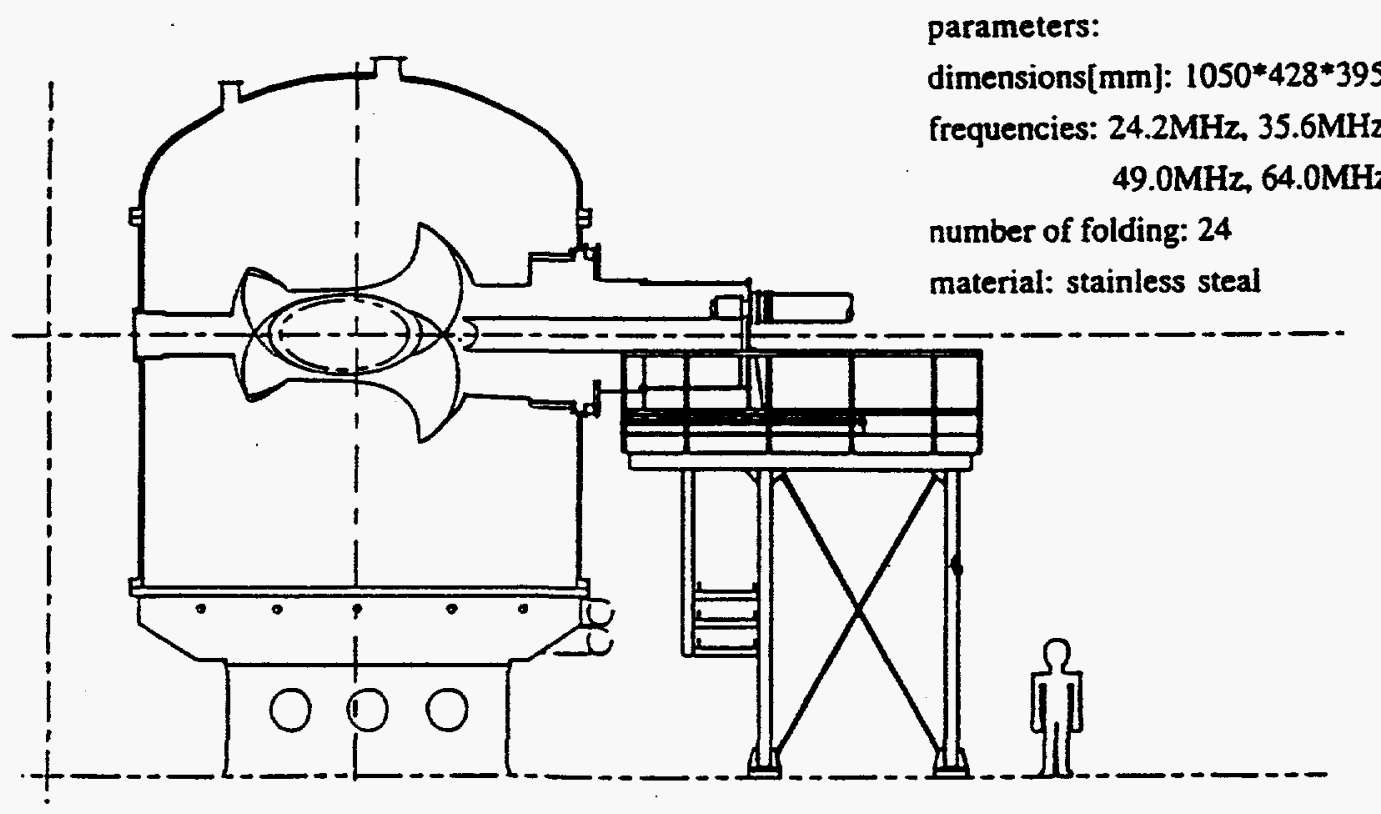

Fig.8 Folded wave guide antenna design for LHD used for plasma production and IBW experiments. 